\title{
Editorial: Physical Review B: Condensed Matter, Then and Now
}

The late Peter Adams, founding editor of Physical Review B (PRB), impishly used to say that the journal was created in 1970 because The Physical Review had reached its binding limit. Apocryphal as that sounds, the birth of PRB couldn't have happened sooner because solid state physics, the core charge of the journal, would soon morph into the broader arena of condensed matter physics (CMP) and then materials physics.

The $125^{\text {th }}$ anniversary of the founding of The Physical Review and the family of journals published by APS offers a chance to look back at some of the landmark publications that have led to PRB becoming not only the largest journal in all of physics but also a venue for excellence.

There is no better place to start than the Nobel Prize-winning pair of papers in 1971 by Wilson, which laid the foundation of the renormalization group theory of phase transitions. These papers not only revolutionized our understanding of scaling and critical phenomena, but soon provided a practical solution of the Kondo problem - the mysterious low-temperature rise in resistivity of a metal with magnetic impurities.

New tools also yielded new insights: The invention of the scanning tunneling microscope in 1981 showed us surfaces at the atomic scale and was a harbinger of the nanophysics revolution of quantum dots, clusters, fullerenes, and nanowires that was to come. In a pair of highly cited papers that appeared in Physical Review Letters and PRB in the early 1980s, Tersoff and Hamann presented the first systematic theoretical study of scanning tunneling microscopy.

The discovery of high-transition-temperature superconductivity in layered cuprates in 1986 set off a frenzy of activity that has few parallels. Theorists responded with new ideas and tools, and experimental techniquesfrom spectroscopy to material synthesis-saw tremendous improvements. One of the most studied models for superconductivity in the cuprates, the $t-J$ model, was introduced by Zhang and Rice in 1988 as a Rapid Communication in PRB. The quest to unravel the physics of the cuprates remains an open and active one.

Technological impact is a hallmark of many PRB papers. Perhaps the fastest advance was the discovery of the giant magnetoresistance (GMR) effect in 1988 by Grünberg (and independently by Fert, published in PRL). GMR-based computer hard disk drives became the norm within a few years, dramatically increasing storage density and speed.

If there is one field that dominates the list of all time top-cited papers in PRB, it is that of density functional approaches to compute the electronic band structure of complex materials. Some of the most influential papers by Kresse, Parr, Perdew, Vanderbilt, and Zunger, among others, were published in the 1980s and 1990s by PRB and have garnered tens of thousands of citations.

PRB has published many influential papers on topological insulators. For example, Yoichi Ando's group in 2010 reported the synthesis of a new bismuth-based 3D topological insulator with the largest surface to bulk conductance ratio. And in 2011, Savrasov and colleagues presented the first proposal for the realization of a Weyl semimetal, a unique state of matter that exhibits topological behavior in both bulk and surface.

But what is the journal's mission now? PRB strives to attract and publish high quality authoritative papers that should stand the test of time. PRB publishes papers in two formats: Regular Articles with no length limit, well suited for a thorough exposition of the research, and Rapid Communications, short letter-size papers for speedy publication of particularly important results. PRB's criteria for publication have remained 
the same: papers should present new and significant understanding and be important to the community in advancing physics.

The role of editors in managing the review process has changed significantly in the last 10 years to become more proactive. Editors now spend more time per paper and have access to more information to make decisions. For some years now, they have been rejecting about a quarter of submissions without external review, based on the quality of the manuscripts and the subject matter fit for the journal. This serves both to speed up the decision-making process and to make more efficient use of referee resources. Editors also often consult our large Editorial Board, our "eyes and ears" in the community, for advice. This helps us make thoughtful, consistent, and fair decisions, and helps authors publish the strongest most useful papers possible.

Since its founding, PRB has grown fourfold. Most of our published content now originates outside the U.S. (in 2017, authors from 70+ countries published their work in PRB). The PRB editorial team is equally international with ties to 15 countries. Our referee pool and Editorial Board are more diverse than ever.

Given this growth, following the literature in CMP outside of one's own subfield can be a challenge. To make it easier, starting in 2008 the editors of PRB have selected a few papers every week for their particular importance, interest, or readability as Editors' Suggestions.

In an era where the pressure to publish in high-profile journals is higher than ever, the one thing that has not changed at PRB over the years is the core value of our mission: to publish solid science minus the hype, vetted in a thorough and professionally run peer review process. In this endeavor, we would like to acknowledge the tireless work of our many thousands of referees, who are the real backbone of our journal.

In the last two decades, there has been an explosion of activity in CMP and PRB has thrived, publishing papers on graphene and other 2D materials, iron-based superconductors, multiferroics, frustrated magnets, metamaterials and nanophotonics, ultracold atoms in optical lattices, quantum spin Hall effect, and Majorana bound states, to cite a few examples. Machine learning, high-throughput computing, and first-principles methods combined with many-body techniques are poised to bring condensed matter and materials physics into a new realm.

With the trust and support of the community, we are excited to see what this journey of scientific discovery will bring in the decades to come.

Sarma Kancharla

Laurens Molenkamp

Published 7 March 2018

DOI: 10.1103/PhysRevB.97.090001

Sarma Kancharla is a PRB Associate Editor and has been working for PRB for 10 years. PRB Lead Editor Laurens Molenkamp is Chair for Experimental Physics at Universität Würzburg.

[This information also appears in the March 2018 issue of APS News aps.org/publications/APSnews.] 